\title{
Computing bounds for the general sum-connectivity index of some graph operations*
}

\author{
S. Akhter and R. Farooq \\ Communicated by D. Simson
}

\begin{abstract}
A BStrACt. Let $G$ be a graph with vertex set $V(G)$ and edge set $E(G)$. Denote by $d_{G}(u)$ the degree of a vertex $u \in V(G)$. The general sum-connectivity index of $G$ is defined as $\chi_{\alpha}(G)=$ $\sum_{u_{1} u_{2} \in E(G)}\left(d_{G}\left(u_{1}\right)+d_{G}\left(u_{2}\right)\right)^{\alpha}$, where $\alpha$ is a real number. In this paper, we compute the bounds for general sum-connectivity index of several graph operations. These operations include corona product, cartesian product, strong product, composition, join, disjunction and symmetric difference of graphs. We apply the obtained results to find the bounds for the general sum-connectivity index of some graphs of general interest.
\end{abstract}

\section{Introduction}

Let $G$ be an undirected, simple, finite and connected graph whose vertex set is $V(G)$ and edge set is $E(G)$. The distance between two vertices $u_{1}$ and $u_{2}$ in $G$, denoted by $d_{G}\left(u_{1}, u_{2}\right)$, is the length of a shortest path between them. For a vertex $u \in V(G), d_{G}(u)$ denotes the degree of $u$. An edge between two vertices $u_{1}$ and $u_{2}$ of $G$ is denoted by $u_{1} u_{2}$. The order and size of $G$ is denoted by $n_{G}$ and $m_{G}$, respectively. The maximum vertex degree of $G$ is denoted by $\triangle_{G}$ and the minimum vertex degree of $G$ is denoted by $\delta_{G}$. A complete graph of order $n$ is denoted by $K_{n}$.

${ }^{*}$ This research is supported by the Higher Education Commission of Pakistan under grant No. 20-3067/NRPU /R\&D/HEC/12.

2010 MSC: 05C76, 05C07.

Key words and phrases: general sum-connectivity index, Randić index, corona product, strong product, symmetric difference. 
A topological index is designed on the ground of transformation of a molecular graph into a number which characterizes the topology of that graph. In chemical graph theory, the degree based topological indices are very important. A topological index, denoted by $\operatorname{Top}(G)$, of a graph $G$ is equal to the topological index $\operatorname{Top}(H)$ of a graph $H$ if and only if $G$ and $H$ are isomorphic.

In the theoretical point of view and applications, the Wiener index is the first and most studied topological index. Initially, Wiener index was known as path index but after some time it was renamed as Wiener index. In 1947, the Wiener index was introduced by the chemist Harold Wiener [18] and is defined as follows:

$$
W(G)=\frac{1}{2} \sum_{u_{1} \in V(G)} \sum_{u_{2} \in V(G)} d_{G}\left(u_{1}, u_{2}\right)
$$

The hyper Wiener index is the extension of Wiener index. The hyper Wiener index $W W(G)$ of a graph $G$ is defined as

$$
W W(G)=\frac{1}{2} \sum_{u \in V(G)} \sum_{v \in V(G)}\left(d_{G}(u, v)+d_{G}(u, v)^{2}\right) .
$$

The Randić index (or product connectivity index) was introduced by Randić [14] in 1975. It is the one of the most used molecular descriptors in structure property and structure activity relationship studies and is defined as follows:

$$
R(G)=\sum_{u_{1} u_{2} \in E(G)} \frac{1}{\sqrt{d_{G}\left(u_{1}\right) d_{G}\left(u_{2}\right)}} .
$$

The general Randić connectivity index (or general product-connectivity index) $R_{\alpha}$ was defined by Kier and Hall [12] in 1976 and is defined as follows:

$$
R_{\alpha}(G)=\sum_{u_{1} u_{2} \in E(G)}\left(d_{G}\left(u_{1}\right) d_{G}\left(u_{2}\right)\right)^{\alpha},
$$

where $\alpha$ is a real number. Then $R_{-1 / 2}$ is the Randic connectivity index. Another variant of the Randic index of $G$ is the harmonic index, denoted by $H(G)$ and is defined as follows:

$$
H(G)=\sum_{a b \in E(G)} \frac{2}{d_{G}(a)+d_{G}(b)}=2 \chi_{-1}(G) .
$$


In 2009, the sum-connectivity index $\chi(G)$ was introduced by Zhou and Trinajestić [19] and is defined as follows:

$$
\chi(G)=\sum_{u_{1} u_{2} \in E(G)} \frac{1}{\sqrt{d_{G}\left(u_{1}\right)+d_{G}\left(u_{2}\right)}} .
$$

A generalized form of the sum-connectivity index was introduced by Zhou and Trinajstić [20] in 2010. It is called general sum-connectivity index $\chi_{\alpha}(G)$ and is defined as:

$$
\chi_{\alpha}(G)=\sum_{u_{1} u_{2} \in E(G)}\left(d_{G}\left(u_{1}\right)+d_{G}\left(u_{2}\right)\right)^{\alpha},
$$

where $\alpha$ is a real number. Then $\chi_{-1 / 2}$ is the sum-connectivity index. The first general Zagreb index of $G$ is introduced by Li and Gutman [13] and is defined as:

$$
M_{1}^{\alpha}(G)=\sum_{u_{1} \in V(G)} d_{G}\left(u_{1}\right)^{\alpha}=\sum_{u_{1} u_{2} \in E(G)}\left(d_{G}\left(u_{1}\right)^{(\alpha-1)}+d_{G}\left(u_{2}\right)^{(\alpha-1)}\right),
$$

where $\alpha$ is a real number with $\alpha \neq 0$ and $\alpha \neq 1$. If $\alpha=2$ then $M_{1}^{2}(G)$ becomes first Zagreb index and if $\alpha=3$ then $M_{1}^{3}(G)$ is called $F$-index. Some chemical graphs that are very interesting in chemical graph theory, can be obtained by the use of different graph operations (graph products). It is important to understand that how the topological indices and topological invariants of such graph operations are related to the topological indices and topological invariants of components of these graph products.

Recently, Khalifeh et al. $[10,11]$ gave the exact expressions for hyper Wiener index and, first and second Zagreb indices of several graph operations. In 2015, Shetty et al. [15] derived formulae for harmonic index of some graph operations. For a detailed study on topological indices of graph operations, we refer to $[2-8,16]$. Very recently, Wang et al. [17] computed the lower and upper bounds for different indices of tricyclic graphs. Gao et al. [9] gave some exact expressions for the hyper-Zagreb index of some graph operations. Akhter et al. [1] gave exact formulae of general sum-connectivity index for some graph operations.

In this paper, we give some bounds for the general sum-connectivity index of several graph operations when $\alpha<0$. These graph operations include corona product, cartesian product, strong product, join, composition, disjunction and symmetric difference of graphs. We apply these results to find the bounds for the general sum-connectivity index of some graphs of general interest. 


\section{Bounds for the general sum-connectivity index}

In this section, we derive some bounds for general sum-connectivity index of several graph operations. Let $G$ and $H$ be two simple connected graphs whose vertex sets are disjoint. For each $u \in V(G)$ and $v \in V(H)$, we have

$$
\begin{array}{cc}
\triangle_{G} \geqslant d_{G}(u), & \delta_{G} \leqslant d_{G}(u), \\
\triangle_{H} \geqslant d_{H}(v), & \delta_{H} \leqslant d_{H}(v) .
\end{array}
$$

The equality holds if and only if $G$ and $H$ are regular graphs.

\subsection{The corona product}

The corona product of $G$ and $H$, denoted by $G \odot H$, is a graph obtained by taking one copy of $G$ and $n_{G}$ copies of $H$ and joining the vertex $u$ that is on $i$-th position in $G$ to every vertex in $i$-th copy of $H$. The order and size of $G \odot H$ are $n_{G}\left(1+n_{H}\right)$ and $m_{G}+n_{G} m_{H}+n_{G} n_{H}$, respectively. The degree of a vertex $u \in V(G \odot H)$ is given by

$$
d_{G \odot H}(u)= \begin{cases}d_{G}(u)+n_{H} & \text { if } u \in V(G) \\ d_{H}(u)+1 & \text { if } u \in V(H)\end{cases}
$$

In the following theorem, the bounds on the general sum-connectivity index of corona product of two graphs are computed.

Theorem 1. Let $\alpha<0$. Then $\beta_{1} \leqslant \chi_{\alpha}(G \odot H) \leqslant \beta_{2}$, where

$$
\begin{aligned}
\beta_{1}=2^{\alpha} & m_{G}\left(\triangle_{G}+n_{H}\right)^{\alpha}+2^{\alpha} n_{G} m_{H}\left(\triangle_{H}+1\right)^{\alpha} \\
& +n_{G} n_{H}\left(\triangle_{G}+\triangle_{H}+n_{H}+1\right)^{\alpha}, \\
\beta_{2}=2^{\alpha} & m_{G}\left(\delta_{G}+n_{H}\right)^{\alpha}+2^{\alpha} n_{G} m_{H}\left(\delta_{H}+1\right)^{\alpha} \\
& +n_{G} n_{H}\left(\delta_{G}+\delta_{H}+n_{H}+1\right)^{\alpha} .
\end{aligned}
$$

The equality holds if and only if $G$ and $H$ are regular graphs. 
Proof. Using (2) and (4) in equation (1), we obtain

$$
\begin{aligned}
\chi_{\alpha}(G \odot H)= & \sum_{u v \in E(G)}\left(d_{G}(u)+d_{G}(v)+2 n_{H}\right)^{\alpha} \\
& +n_{G} \sum_{u v \in E(H)}\left(d_{H}(u)+d_{H}(v)+2\right)^{\alpha} \\
& +\sum_{u \in V(G)} \sum_{v \in V(H)}\left(d_{G}(u)+n_{H}+d_{H}(v)+1\right)^{\alpha} \\
\geqslant & \sum_{u v \in E(G)}\left(2 \triangle_{G}+2 n_{H}\right)^{\alpha}+n_{G} \sum_{u v \in E(H)}\left(2 \triangle_{H}+2\right)^{\alpha} \\
& +\sum_{u \in V(G)} \sum_{v \in V(H)}\left(\triangle_{G}+n_{H}+\triangle_{H}+1\right)^{\alpha} \\
= & 2^{\alpha} m_{G}\left(\triangle_{G}+n_{H}\right)^{\alpha}+2^{\alpha} n_{G} m_{H}\left(\triangle_{H}+1\right)^{\alpha} \\
& \quad+n_{G} n_{H}\left(\triangle_{G}+\triangle_{H}+n_{H}+1\right)^{\alpha} .
\end{aligned}
$$

Similarly, we can compute

$$
\begin{gathered}
\chi_{\alpha}(G \odot H) \leqslant 2^{\alpha} m_{G}\left(\delta_{G}+n_{H}\right)^{\alpha}+2^{\alpha} n_{G} m_{H}\left(\delta_{H}+1\right)^{\alpha} \\
+n_{G} n_{H}\left(\delta_{G}+\delta_{H}+n_{H}+1\right)^{\alpha} .
\end{gathered}
$$

The equality in (5) and (6) holds if and only if $G$ and $H$ are regular graphs.

Let $t \geqslant 1$ and $\overline{K_{t}}$ be the complement of $K_{t}$. Then $t$-thorny graph of $G$ is the corona product of $G$ and $\overline{K_{t}}$. The following corollary is an easy consequence of Theorem 1 .

Corollary 1. For $\alpha<0$, the following holds:

$$
\begin{gathered}
2^{\alpha} m_{G}\left(\triangle_{G}+t\right)+n_{G} t\left(\triangle_{G}+t+1\right) \leqslant \chi_{\alpha}\left(G \odot \overline{K_{t}}\right) \\
\leqslant 2^{\alpha} m_{G}\left(\delta_{G}+t\right)+n_{G} t\left(\delta_{G}+t+1\right) .
\end{gathered}
$$

\subsection{The cartesian product}

The cartesian product of $G$ and $H$, denoted by $G \square H$, is a graph whose vertex set is $V(G \square H)=V(G) \times V(H)$ and two vertices $\left(u_{1}, v_{1}\right)$ and $\left(u_{2}, v_{2}\right)$ are adjacent in $G \square H$ whenever $\left[v_{1}\right.$ and $v_{2}$ are adjacent in $H$ and $\left.u_{1}=u_{2}\right]$ or $\left[u_{1}\right.$ and $u_{2}$ are adjacent in $G$ and $\left.v_{1}=v_{2}\right]$. The order of the cartesian product of two graphs is the product of number of vertices of $G$ and $H$, and the size is $m_{G} n_{H}+m_{H} n_{G}$. If $G$ and $H$ are regular graphs 
then $G \square H$ is also regular graph. The degree of a vertex $(u, v) \in V(G \square H)$ is

$$
d_{G \square H}((u, v))=d_{G}(u)+d_{H}(v) .
$$

In the following theorem, we compute bounds on the general sum- connectivity index of $G \square H$.

Theorem 2. Let $\alpha<0$. Then $\beta_{1} \leqslant \chi_{\alpha}(G \square H) \leqslant \beta_{2}$, where

$$
\beta_{1}=2^{\alpha} m_{G \square H}\left(\triangle_{G}+\triangle_{H}\right)^{\alpha}, \beta_{2}=2^{\alpha} m_{G \square H}\left(\delta_{G}+\delta_{H}\right)^{\alpha} .
$$

The equality holds if and only if $G$ and $H$ are regular graphs.

Proof. Using (2) and (7) in equation (1), we obtain

$$
\begin{aligned}
\chi_{\alpha}(G \square H)= & \sum_{u_{1} \in V(G)} \sum_{v_{1} v_{2} \in E(H)}\left(2 d_{G}\left(u_{1}\right)+d_{H}\left(v_{1}\right)+d_{H}\left(v_{2}\right)\right)^{\alpha} \\
& +\sum_{v_{1} \in V(H)} \sum_{u_{1} u_{2} \in E(G)}\left(d_{G}\left(u_{1}\right)+d_{G}\left(u_{2}\right)+2 d_{H}\left(v_{1}\right)\right)^{\alpha} \\
\geqslant & \sum_{u_{1} \in V(G)} \sum_{v_{1} v_{2} \in E(H)} 2^{\alpha}\left(\triangle_{G}+\triangle_{H}\right)^{\alpha} \\
& +\sum_{v_{1} \in V(H)} \sum_{u_{1} u_{2} \in E(G)} 2^{\alpha}\left(\triangle_{G}+\triangle_{H}\right)^{\alpha} \\
= & 2^{\alpha}\left(n_{G} m_{H}+n_{H} m_{G}\right)\left(\triangle_{G}+\triangle_{H}\right)^{\alpha} \\
= & 2^{\alpha} m_{G \square H}\left(\triangle_{G}+\triangle_{H}\right)^{\alpha} .
\end{aligned}
$$

One can analogously compute the following for $\alpha<0$ :

$$
\chi_{\alpha}(G \square H) \leqslant 2^{\alpha} m_{G \square H}\left(\delta_{G}+\delta_{H}\right)^{\alpha} .
$$

The equality in (8) and (9) obviously holds if and only if $G$ and $H$ are regular graphs.

\subsection{The strong product}

The strong product of $G$ and $H$, denoted by $G \otimes H$, is a graph whose vertex set is $V(G \otimes H)=V(G) \times V(H)$ and two vertices $\left(u_{1}, v_{1}\right)$ and $\left(u_{2}, v_{2}\right)$ are adjacent in $G \otimes H$ whenever $\left[v_{1}\right.$ and $v_{2}$ are adjacent in $H$ and $\left.u_{1}=u_{2}\right]$ or $\left[u_{1}\right.$ and $u_{2}$ are adjacent in $G$ and $\left.v_{1}=v_{2}\right]$ or $\left[u_{1} u_{2} \in E(G)\right.$ and $\left.v_{1} v_{2} \in E(H)\right]$. The order of $G \otimes H$ is the product of number of vertices 
of $G$ and $H$, and the size is $n_{G} m_{H}+n_{H} m_{G}+2 m_{G} m_{H}$. The degree of a vertex $(u, v) \in V(G \otimes H)$ is

$$
d_{G \otimes H}((u, v))=d_{G}(u)+d_{H}(v)+d_{G}(u) d_{H}(v) .
$$

We compute bounds on the general sum-connectivity index of $G \otimes H$ in the following theorem.

Theorem 3. Let $\alpha<0$. Then $\beta_{1} \leqslant \chi_{\alpha}(G \otimes H) \leqslant \beta_{2}$, where

$$
\begin{aligned}
& \beta_{1}=2^{\alpha} m_{G \bowtie H}\left(\triangle_{G}+\triangle_{H}+\triangle_{G} \triangle_{H}\right)^{\alpha}, \\
& \beta_{2}=2^{\alpha} m_{G \unrhd H}\left(\delta_{G}+\delta_{H}+\delta_{G} \delta_{H}\right)^{\alpha} .
\end{aligned}
$$

The equality holds if and only if $G$ and $H$ are regular graphs.

Proof. Using (2) and (10) in equation (1), we obtain

$$
\begin{aligned}
\chi_{\alpha}(G \otimes H)= & \sum_{u_{1} \in V(G)} \sum_{v_{1} v_{2} \in E(H)}\left(2 d_{G}\left(u_{1}\right)+\left(d_{H}\left(v_{1}\right)+d_{H}\left(v_{2}\right)\right)\right. \\
& \left.+d_{G}\left(u_{1}\right)\left(d_{H}\left(v_{1}\right)+d_{H}\left(v_{2}\right)\right)\right)^{\alpha} \\
& +\sum_{v_{1} \in V(H)} \sum_{u_{1} u_{2} \in E(G)}\left(\left(d_{G}\left(u_{1}\right)+d_{G}\left(u_{2}\right)\right)+2 d_{H}\left(v_{1}\right)\right. \\
& \left.+d_{H}\left(v_{1}\right)\left(d_{G}\left(u_{1}\right)+d_{G}\left(u_{2}\right)\right)\right)^{\alpha} \\
& +2 \sum_{u_{1} u_{2} \in E(G)} \sum_{v_{1} v_{2} \in E(H)}\left(\left(d_{G}\left(u_{1}\right)+d_{G}\left(u_{2}\right)\right)+\left(d_{H}\left(v_{1}\right)\right.\right. \\
& \left.\left.\left.+d_{H}\left(v_{2}\right)\right)+d_{G}\left(u_{1}\right) d_{H}\left(v_{1}\right)+d_{G}\left(u_{2}\right) d_{H}\left(v_{2}\right)\right)\right)^{\alpha} \\
\geqslant & \sum_{u_{1} \in V(G)} \sum_{v_{1} v_{2} \in E(H)} 2^{\alpha}\left(\triangle_{G}+\triangle_{H}+\triangle_{G} \triangle_{H}\right)^{\alpha} \\
& +\sum_{v_{1} \in V(H)} \sum_{u_{1} u_{2} \in E(G)} 2^{\alpha}\left(\triangle_{G}+\triangle_{H}+\triangle_{G} \triangle_{H}\right)^{\alpha} \\
& +2 \sum_{u_{1} u_{2} \in E(G)} \sum_{v_{1} v_{2} \in E(H)} 2^{\alpha}\left(\triangle_{G}+\triangle_{H}+\triangle_{G} \triangle_{H}\right)^{\alpha} \\
= & 2^{\alpha}\left(n_{G} m_{H}+n_{H} m_{G}+2 m_{G} m_{H}\right)\left(\triangle_{G}+\triangle_{H}+\triangle_{G} \triangle_{H}\right)^{\alpha} \\
= & 2^{\alpha} m_{G \otimes H}\left(\triangle_{G}+\triangle_{H}+\triangle_{G} \triangle_{H}\right)^{\alpha} .
\end{aligned}
$$

Analogously, one can compute the following:

$$
\chi_{\alpha}(G \otimes H) \leqslant 2^{\alpha} m_{G \otimes H}\left(\delta_{G}+\delta_{H}+\delta_{G} \delta_{H}\right)^{\alpha} .
$$

If $G$ and $H$ are regular graphs then the equality in (11) and (12) holds. 


\subsection{The join}

The join of $G$ and $H$, denoted by $G+H$, is a union of graphs $G$ and $H$ together with all the edges joining the sets of vertices of $G$ and $H$. The order and size of $G+H$ are $n_{G} n_{H}$ and $m_{G}+m_{H}+n_{G} n_{H}$, respectively. The degree of a vertex $u$ in $G+H$ is given by

$$
d_{G+H}(u)= \begin{cases}d_{G}(u)+n_{H} & \text { if } u \in V(G) \\ d_{H}(u)+n_{G} & \text { if } u \in V(H) .\end{cases}
$$

We compute bounds on the general sum-connectivity index for join of two graphs in the following theorem.

Theorem 4. Let $\alpha<0$. Then $\beta_{1} \leqslant \chi_{\alpha}(G+H) \leqslant \beta_{2}$, where

$$
\begin{gathered}
\beta_{1}=2^{\alpha} m_{G}\left(\triangle_{G}+n_{H}\right)^{\alpha}+2^{\alpha} m_{H}\left(\triangle_{H}+n_{G}\right)^{\alpha} \\
+n_{G} n_{H}\left(\triangle_{G}+\triangle_{H}+n_{G}+n_{H}\right)^{\alpha}, \\
\beta_{2}=2^{\alpha} m_{G}\left(\delta_{G}+n_{H}\right)^{\alpha}+2^{\alpha} m_{H}\left(\delta_{H}+n_{G}\right)^{\alpha} \\
+n_{G} n_{H}\left(\delta_{G}+\delta_{H}+n_{G}+n_{H}\right)^{\alpha} .
\end{gathered}
$$

The equality holds if and only if $G$ and $H$ are regular graphs.

Proof. Using (2) and (13) in equation (1), we get

$$
\begin{aligned}
\chi_{\alpha}(G+H)= & \sum_{u v \in E(G)}\left(d_{G}(u)+d_{G}(v)+2 n_{H}\right)^{\alpha} \\
& +\sum_{u v \in E(H)}\left(d_{H}(u)+d_{H}(v)+2 n_{G}\right)^{\alpha} \\
& +\sum_{u \in V(G)} \sum_{v \in V(H)}\left(d_{G}(u)+n_{H}+d_{H}(v)+n_{G}\right)^{\alpha} \\
\geqslant & \sum_{u v \in E(G)} 2^{\alpha}\left(\triangle_{G}+n_{H}\right)^{\alpha}+\sum_{a b \in E(H)} 2^{\alpha}\left(\triangle_{H}+n_{G}\right)^{\alpha} \\
& +\sum_{u \in V(G)} \sum_{v \in V(H)}\left(\triangle_{G}+\triangle_{H}+n_{G}+n_{H}\right)^{\alpha} \\
= & 2^{\alpha} m_{G}\left(\triangle_{G}+n_{H}\right)^{\alpha}+2^{\alpha} m_{H}\left(\triangle_{H}+n_{G}\right)^{\alpha} \\
+ & n_{G} n_{H}\left(\triangle_{G}+\triangle_{H}+n_{G}+n_{H}\right)^{\alpha} .
\end{aligned}
$$

Similarly, we can show that

$$
\begin{gathered}
\chi_{\alpha}(G+H) \leqslant 2^{\alpha} m_{G}\left(\delta_{G}+n_{H}\right)^{\alpha}+2^{\alpha} m_{H}\left(\delta_{H}+n_{G}\right)^{\alpha} \\
+n_{G} n_{H}\left(\delta_{G}+\delta_{H}+n_{G}+n_{H}\right)^{\alpha} .
\end{gathered}
$$

If $G$ and $H$ are regular graphs then we obtain the equality in (14) and (15). 


\subsection{The composition}

The composition or lexicographic product of $G$ and $H$, denoted by $G[H]$, is the graph whose vertex set is $V(G[H])=V(G) \times V(H)$ and two vertices $\left(u_{1}, v_{1}\right)$ and $\left(u_{2}, v_{2}\right)$ are adjacent in $G[H]$ whenever $\left[u_{1} u_{2} \in E(G)\right]$ or $\left[v_{1}\right.$ and $v_{2}$ are adjacent in $H$ and $\left.u_{1}=u_{2}\right]$. The order of $G[H]$ is the product of number of vertices of $G$ and $H$, and size is $m_{G} n_{H}^{2}+n_{G} m_{H}$. The degree of a vertex $(u, v) \in V(G[H])$ is

$$
d_{G[H]}((u, v))=n_{H} d_{G}(u)+d_{H}(v) .
$$

In the following theorem, we calculate bounds on the general sum- connectivity index for composition of two graphs.

Theorem 5. Let $\alpha<0$. Then $\beta_{1} \leqslant \chi_{\alpha}(G[H]) \leqslant \beta_{2}$, where

$$
\beta_{1}=2^{\alpha} m_{G[H]}\left(n_{H} \triangle_{G}+\triangle_{H}\right)^{\alpha}, \beta_{2}=2^{\alpha} m_{G[H]}\left(n_{H} \delta_{G}+\delta_{H}\right)^{\alpha} .
$$

The equality holds if and only if $G$ and $H$ are regular graphs.

Proof. Using (2) and (16) in equation (1), we obtain

$$
\begin{aligned}
\chi_{\alpha}(G[H])= & \sum_{u_{1} \in V(G)} \sum_{v_{1} v_{2} \in E(H)}\left(2 n_{H} d_{G}\left(u_{1}\right)+d_{H}\left(v_{1}\right)+d_{H}\left(v_{2}\right)\right)^{\alpha} \\
& +\sum_{v_{1} \in V(H)} \sum_{v_{2} \in V(H)} \sum_{u_{1} u_{2} \in E(G)}\left(n_{H}\left(d_{G}\left(u_{1}\right)+d_{G}\left(u_{2}\right)\right)\right. \\
& \left.+d_{H}\left(v_{1}\right)+d_{H}\left(v_{2}\right)\right)^{\alpha} \\
\geqslant & \sum_{u_{1} \in V(G)} \sum_{v_{1} v_{2} \in E(H)} 2^{\alpha}\left(n_{H} \triangle_{G}+\triangle_{H}\right)^{\alpha} \\
& \quad+\sum_{v_{1} \in V(H)} \sum_{v_{2} \in V(H)} \sum_{u_{1} u_{2} \in E(G)} 2^{\alpha}\left(n_{H} \triangle_{G}+\triangle_{H}\right)^{\alpha} \\
= & 2^{\alpha}\left(n_{G} m_{H}+n_{H}^{2} m_{G}\right)\left(n_{H} \triangle_{G}+\triangle_{H}\right)^{\alpha} \\
= & 2^{\alpha} m_{G[H]}\left(n_{H} \triangle_{G}+\triangle_{H}\right)^{\alpha} .
\end{aligned}
$$

Analogously, one can compute the upper bound

$$
\chi_{\alpha}(G[H]) \leqslant 2^{\alpha} m_{G[H]}\left(n_{H} \delta_{G}+\delta_{H}\right)^{\alpha} .
$$

The equality in (17) and (18) obviously holds if and only if $G$ and $H$ are regular graphs. 


\subsection{The disjunction}

The disjunction of $G$ and $H$, denoted by $G \vee H$, is a graph whose vertex set is $V(G \vee H)=V(G) \times V(H)$ and two vertices $\left(u_{1}, v_{1}\right)$ and $\left(u_{2}, v_{2}\right)$ are adjacent in $G \vee H$ whenever $\left[v_{1} v_{2} \in E(H)\right]$ or $\left[u_{1} u_{2} \in E(G)\right]$. The order of $G \vee H$ is the product of number of vertices of graphs $G$ and $H$, and size is $m_{G} n_{H}^{2}+m_{H} n_{G}^{2}-2 m_{G} m_{H}$. The degree of a vertex $(u, v) \in G \vee H$ is

$$
d_{G \vee H}((u, v))=n_{H} d_{G}(u)+n_{G} d_{H}(v)-d_{G}(u) d_{H}(v) .
$$

In the following theorem, we compute lower and upper bounds on the general sum-connectivity index for $G \vee H$.

Theorem 6. Let $\alpha<0$. Then $\beta_{1} \leqslant \chi_{\alpha}(G \vee H) \leqslant \beta_{2}$, where

$$
\beta_{1}=2^{\alpha} m_{G \vee H}\left(n_{H} \triangle_{G}+n_{G} \triangle_{H}\right)^{\alpha}, \beta_{2}=2^{\alpha} m_{G \vee H}\left(n_{H} \delta_{G}+n_{G} \delta_{H}\right)^{\alpha} .
$$

The equality holds if and only if $G$ and $H$ are regular graphs.

Proof. Using (2) and (19) in equation (1), we obtain

$$
\begin{aligned}
\chi_{\alpha}(G & \vee H)=\sum_{u_{1} \in V_{1}} \sum_{u_{2} \in V(G)} \sum_{v_{1} v_{2} \in E(H)}\left(n_{H}\left(d_{G}\left(u_{1}\right)+d_{G}\left(u_{2}\right)\right)+n_{G}\left(d_{H}\left(v_{1}\right)\right.\right. \\
& \left.\left.+d_{H}\left(v_{2}\right)\right)-\left(d_{G}\left(u_{1}\right) d_{H}\left(v_{1}\right)+d_{G}\left(u_{2}\right) d_{H}\left(v_{2}\right)\right)\right)^{\alpha} \\
& +\sum_{v_{1} \in V(H)} \sum_{v_{2} \in V(H)} \sum_{u_{1} u_{2} \in E(G)}\left(n_{H}\left(d_{G}\left(u_{1}\right)+d_{G}\left(u_{2}\right)\right)+n_{G}\left(d_{H}\left(v_{1}\right)\right.\right. \\
& \left.\left.+d_{H}\left(v_{2}\right)\right)-\left(d_{G}\left(u_{1}\right) d_{H}\left(v_{1}\right)+d_{G}\left(u_{2}\right) d_{H}\left(v_{2}\right)\right)\right)^{\alpha} \\
& -2 \sum_{u_{1} u_{2} \in E(G)} \sum_{v_{1} v_{2} \in E(H)}\left(n_{H}\left(d_{G}\left(u_{1}\right)+d_{G}\left(u_{2}\right)\right)+n_{G}\left(d_{H}\left(v_{1}\right)\right.\right. \\
& \left.\left.+d_{H}\left(v_{2}\right)\right)-\left(d_{G}\left(u_{1}\right) d_{H}\left(v_{1}\right)+d_{G}\left(u_{2}\right) d_{H}\left(v_{2}\right)\right)\right)^{\alpha} \\
\geqslant & \sum_{u_{1} \in V(G)} \sum_{u_{2} \in V(G)} \sum_{v_{1} v_{2} \in E(H)} 2^{\alpha}\left(n_{H} \triangle_{G}+n_{G} \triangle_{H}-\triangle_{G} \triangle_{H}\right)^{\alpha} \\
& +\sum_{v_{1} \in V(H)} \sum_{v_{2} \in V(H)} \sum_{u_{1} u_{2} \in E(G)} 2^{\alpha}\left(n_{H} \triangle_{G}+n_{G} \triangle_{H}-\triangle_{G} \triangle_{H}\right)^{\alpha} \\
& -2 \sum_{u_{1} u_{2} \in E(G)} \sum_{v_{1} v_{2} \in E(H)} 2^{\alpha}\left(n_{H} \triangle_{G}+n_{G} \triangle_{H}-\triangle_{G} \triangle_{H}\right)^{\alpha} \\
= & 2^{\alpha}\left(n_{G} m_{H}+n_{H} m_{G}-2 m_{G} m_{H}\right)\left(n_{H} \triangle_{G}+n_{G} \triangle_{H}-\triangle_{G} \triangle_{H}\right)^{\alpha} \\
= & 2^{\alpha} m_{G \vee H}\left(n_{H} \triangle_{G}+n_{G} \triangle_{H}\right)^{\alpha} .
\end{aligned}
$$


Similarly, we can compute,

$$
\chi_{\alpha}(G \vee H) \leqslant 2^{\alpha} m_{G \vee H}\left(n_{H} \delta_{G}+n_{G} \delta_{H}\right)^{\alpha} .
$$

The equality in (20) and (21) obviously holds if and only if $G$ and $H$ are regular.

\subsection{The symmetric difference}

The symmetric difference of $G$ and $H$, denoted by $G \oplus H$, is a graph whose vertex set is $V(G \oplus H)=V(G) \times V(H)$ and two vertices $\left(u_{1}, v_{1}\right)$ and $\left(u_{2}, v_{2}\right)$ are adjacent in $G \oplus H$ whenever $\left[u_{1} u_{2} \in E(G)\right]$ or $\left[v_{1} v_{2} \in E(H)\right]$ but not both. The order of $G \oplus H$ is the product of number of vertices graphs of $G$ and $H$, and size is $m_{G} n_{H}^{2}+m_{H} n_{G}^{2}-4 m_{G} m_{H}$. The degree of a vertex $(u, v) \in V(G \oplus H)$ is $d_{G \oplus H}((u, v))=n_{H} d_{G}(u)+n_{G} d_{H}(v)-$ $2 d_{G}(u) d_{H}(v)$. In the following theorem, we compute bounds for the general sum-connectivity index for symmetric difference of two graphs. The proof is similar to the proof of Theorem 6 , hence omitted.

Theorem 7. Let $\alpha<0$. Then $\beta_{1} \leqslant \chi_{\alpha}(G \oplus H) \leqslant \beta_{2}$, where

$$
\begin{aligned}
& \beta_{1}=2^{\alpha} m_{G \oplus H}\left(n_{H} \triangle_{G}+n_{G} \triangle_{H}-2 \triangle_{G} \triangle_{H}\right)^{\alpha}, \\
& \beta_{2}=2^{\alpha} m_{G \oplus H}\left(n_{H} \delta_{G}+n_{G} \delta_{H}-2 \delta_{G} \delta_{H}\right)^{\alpha} .
\end{aligned}
$$

The equality holds if and only if $G$ and $H$ are regular graphs.

Remark. In Theorems 1-7, we assumed that $\alpha<0$. However, if $\alpha>0$ then all inequalities in Theorems $1-7$ will be reversed.

\section{Few examples}

A path $P_{n}$ of length $n-1$ is a graph with vertex set $\left\{u_{i} \mid i=1, \ldots, n\right\}$ and edge set $\left\{u_{i} u_{i+1} \mid i=1, \ldots, n-1\right\}$. A cycle $C_{n}$ of length $n$ is a graph with vertex set $\left\{u_{i} \mid i=1, \ldots, n\right\}$ and edge set $\left\{u_{i} u_{i+1} \mid i=\right.$ $1, \ldots, n-1\} \cup\left\{u_{n} u_{1}\right\}$. In this section, we apply the results of Section 1 to find bounds of some particular graphs.

The bottleneck graph, denoted by $B$, of a graph $G$ is the hydrogen suppressed molecular graph. It is defined as $B=K_{2} \odot G$. Let $G_{1}, G_{2}, \ldots, G_{n}$ be the pairwise disjoint graphs. The bridge graph with respect to the vertices $v_{j} \in G_{j}$ where $j=1,2, \ldots, n$, denoted by $B\left(G_{1}, G_{2}, . ., G_{n}, v_{1}, v_{2}, . ., v_{n}\right)$ is the graph obtained by connecting the vertices $v_{j} \in V\left(G_{j}\right)$ and $v_{j+1} \in$ $V\left(G_{j+1}\right)$ by an edge $v_{j} v_{j+1}$, where $j=1,2, \ldots, n$. Taking $n$ copies of 
the graph $G, n \geqslant 2$, and a vertex $v \in V(G)$, a special case of the bridge graph is defined by $G_{n}(G, v)=B(G, G, \ldots, G, v, v, v, \ldots, v)$. Note that $G_{1}(G, v)=G$ for any vertex $v \in V(G)$. Let $v$ be a vertex of $P_{3}$ of degree 2. Then it is known that $G_{n}\left(P_{3}, v\right) \cong P_{n} \odot \overline{K_{2}}$. Also, for any $u \in V\left(C_{k}\right)$, we know that $G_{n}\left(C_{k}, u\right) \cong P_{n} \odot K_{2}$. Using Theorem 1, the upper and lower bounds on the general sum-connectivity index of $B, P_{n} \odot \overline{K_{2}}$ and $P_{n} \odot K_{2}$ for $n \geqslant 2$ are as follows:

$$
\begin{gathered}
2^{\alpha}\left(n_{G}+1\right)^{\alpha}+2^{\alpha+1} m_{G}\left(\triangle_{G}+1\right)^{\alpha}+2 n_{G}\left(\triangle_{G}+n+2\right)^{\alpha} \\
\leqslant \chi_{\alpha}(B) \leqslant 2^{\alpha}\left(n_{G}+1\right)^{\alpha}+2^{\alpha+1} m_{G}\left(\delta_{G}+1\right)^{\alpha}+2 n_{G}\left(\delta_{G}+n+2\right)^{\alpha}, \\
2^{\alpha} n\left(4^{\alpha}+2 \times 5^{\alpha}\right)-8^{\alpha} \leqslant \chi_{\alpha}\left(P_{n} \odot \overline{K_{2}}\right) \leqslant 2^{\alpha} n\left(3^{\alpha}+2^{\alpha+1}\right)-6^{\alpha}, \\
2^{\alpha} n\left(4^{\alpha}+2^{\alpha}+2 \times 3^{\alpha}\right)-8^{\alpha} \leqslant \chi_{\alpha}\left(P_{n} \odot K_{2}\right) \leqslant \\
n\left(6^{\alpha}+4^{\alpha}+2 \times 5^{\alpha}\right)-6^{\alpha} .
\end{gathered}
$$

The graph $G=P_{n} \square C_{m}$ and $S=C_{n} \square C_{m}$ denote a nanotube and nanotrous, respectively, where $m \geqslant 3$ and $n \geqslant 2$. Using Theorem 2, we can compute bounds on the general sum-connectivity index of nanotube $G$ as follows:

$$
m(2 n-1) 8^{\alpha} \leqslant \chi_{\alpha}(G) \leqslant m(2 n-1) 6^{\alpha} .
$$

The nanotrous $S$ is a regular graph. Using Theorem 2, we can compute the exact formula for the general sum-connectivity index of $S$ as follows:

$$
\chi_{\alpha}(S)=2^{3 \alpha+1} m n .
$$

The suspension of a graph $G$, denoted by $K_{1}+G$, is the join of $K_{1}$ and $G$. The complete bipartite graph $K_{n_{1}, n_{2}}$ is defined as $\overline{K_{n_{1}}}+\overline{K_{n_{2}}}$. The wheel graph is defined by $W_{n}=K_{1}+C_{n}$ and cone graph is defined by $C_{n_{1}, n_{2}}=C_{n_{1}}+\overline{K_{n_{2}}}$. Using Theorem 4 , we can compute the bounds on the general sum-connectivity index of $K_{1}+G$ as follows:

$$
\begin{aligned}
& 2^{\alpha} m_{G}\left(\triangle_{G}+1\right)^{\alpha}+n_{G}\left(\triangle_{G}+n_{G}+1\right)^{\alpha} \\
& \quad \leqslant \chi_{\alpha}\left(K_{1}+G\right) \leqslant 2^{\alpha} m_{G}\left(\delta_{G}+1\right)^{\alpha}+n_{G}\left(\delta_{G}+n_{G}+1\right)^{\alpha} .
\end{aligned}
$$

Using Theorem 4, we can compute the exact formulae for the general sum-connectivity index of $K_{n_{1}, n_{2}}, W_{n}$ and $C_{n_{1}, n_{2}}$ as follows:

$$
\begin{aligned}
\chi_{\alpha}\left(K_{n_{1}, n_{2}}\right) & =n_{1} n_{2}\left(n_{1}+n_{2}\right)^{\alpha}, \\
\chi_{\alpha}\left(W_{n}\right) & =6^{\alpha} n+n(n+3)^{\alpha}, \\
\chi_{\alpha}\left(C_{n_{1}, n_{2}}\right) & =2^{\alpha} n_{1}\left(n_{2}+2\right)^{\alpha}+n_{1} n_{2}\left(n_{1}+n_{2}+2\right)^{\alpha} .
\end{aligned}
$$


The fence graph, denoted by $P_{n}\left[P_{2}\right]$, is the composition of $P_{n}$ and $P_{2}$. Using Theorem 5, we can compute the bounds on the general sumconnectivity index of $P_{n}\left[P_{m}\right]$.

$$
10^{\alpha}(5 n-4) \leqslant \chi_{\alpha}\left(P_{n}\left[P_{2}\right]\right) \leqslant 6^{\alpha}(5 n-4) .
$$

The closed fence graph, denoted by $C_{n}\left[P_{2}\right]$, is the composition of $C_{n}$ and $P_{2}$. The graph $C_{n}\left[P_{2}\right]$ is a regular graph. Using Theorem 5 , we can compute the exact formula for the general sum-connectivity index of $C_{n}\left[P_{2}\right]$ as follows:

$$
\chi_{\alpha}\left(C_{n}\left[P_{2}\right]\right)=5 \times 10^{\alpha} n .
$$

By using Theorems 3, 6 and 7, the bounds on the general sumconnectivity index of $P_{n} \otimes P_{m}, P_{n} \vee P_{m}$ and $P_{n} \oplus P_{m}$ are given below:

1) $4^{2 \alpha}(4 m n-3 m-3 n+2) \leqslant \chi_{\alpha}\left(P_{n} \otimes P_{m}\right) \leqslant 6^{\alpha}(4 m n-3 m-3 n+2)$,

2) $4^{\alpha}[m n(m+n-2)+m(2-m)+n(2-n)-2](m+n-2)^{\alpha} \leqslant$ $\chi_{\alpha}\left(P_{n} \vee P_{m}\right) \leqslant 4^{\alpha}[m n(m+n-2)+m(2-m)+n(2-n)-2](m+n-2)^{\alpha}$, 3) $4^{\alpha}[m n(m+n-4)+m(4-m)+n(4-n)-4](m+n-4)^{\alpha} \leqslant$ $\chi_{\alpha}\left(P_{n} \oplus P_{m}\right) \leqslant 2^{\alpha}[m n(m+n-4)+m(4-m)+n(4-n)-4](m+n-2)^{\alpha}$.

\section{References}

[1] S. Akhter, R. Farooq, S. Pirzada, Exact formulae of general sum-connectivity index for some graph operations, Matematički Vesnik, 70(3), 267-282 (2018).

[2] A. R. Ashrafi, T. Doslic and A. Hamzeh, The Zagreb coindices of graph operations, Discrete Appl. Math., 158, 1571-1578 (2010).

[3] M. Azari, Sharp lower bounds on the Narumi-Katayama index of graph operations, Appl. Math. Comput., 239, 409-421 (2014).

[4] M. Azari, A. Iranmanesh, Computing the eccentric-distance sum for graph operations, Discrete. Appl. Math., 161(18), 2827-2840 (2013).

[5] M. Azari and A. Iranmanesh, Some inequalities for the multiplicative sum Zagreb index of graph operations, J. Math. Inequal., 9(3), 727-738 (2015).

[6] N. De, S. M. A. Nayeem, A. Pal, F-index of some graph operations, Discrete Mathematics, Algorithms and Applications, 8(2), 1650025 (2016).

[7] M. Eliasi, G. Raeisi, B. Taeri, Wiener index of some graph operations, Discrete Appl. Math., 160, 1333-1344 (2012).

[8] B. Eskender and E. Vumar, Eccentric connectivity index and eccentric distance sum of some graph operations, Trans. Comb., 2(1), 103-111 (2013).

[9] W. Gao, M. K. Jamil, M. R. Farahani, The hyper-Zagreb index and some graph operations, J. Appl. Math. Comput., 54(1), 263-275 (2017).

[10] M. H. Khalifeh, A. R. Ashrafi, H. Y. Azari, The first and second Zagreb indices of some graphs operations, Discrete Appl. Math., 157, 804-811 (2009). 
[11] M. H. Khalifeh, H. Y. Azari, A. R. Ashrafi, The hyper-Wiener index of graph operations, Comput. Math. Appl., 56, 1402-1407 (2008).

[12] L. B. Kier, L. H. Hall, Molecular Connectivity in Chemistry and Drug Research, Acad. Press, (1976).

[13] X. Li, I. Gutman, Mathematical aspects of Randić type molecular structure descriptors, Univ. Kragujevac, Kragujevac, (2006).

[14] M. Randić, On characterization of molecular branching, J. Am. Chem. Soc., 97, 6609-6615 (1975).

[15] B. S. Shetty, V. Lokesha, P. S. Ranjini, On the harmonic index of graph operations, Transactions on Combinatorics, 4(4), 5-14 (2015).

[16] M. Veylaki, M. J. Nikmehr and H. A. Tavallaee, The third and hyper-Zagreb coindices of some graph operations, J. Appl. Math. Comput., 50(1), 315-325 (2016).

[17] D. Wang, S. Tan, L. Zhu, On the lower and upper bounds for different indices of tricyclic graphs, J. Appl. Math. Comput., 51, 1-11 (2016).

[18] H. Wiener, Structural determination of the paraffin boiling points, J. Am. Chem. Soc., 69, 17-20 (1947).

[19] B. Zhou, N. Trinajstić, On a novel connectivity index, J. Math. Chem., 46, 12521270 (2009).

[20] B. Zhou, N. Trinajstić, On general sum-connectivity index, J. Math. Chem., 47, 210-218 (2010).

\section{CONTACT INFORMATION}

Shehnaz Akhter, School of Natural Sciences, National University Rashid Farooq of Sciences and Technology, H-12 Islamabad, Pakistan

$$
\text { E-Mail(s): shehnazakhter36@yahoo.com, }
$$
farook.ra@gmail.com

Received by the editors: 15.08.2016. 